\title{
Research on the Interactive Response Relationship between Price and Load Based on Market Elasticity
}

\author{
Pengcheng Zang, Zhijun $\mathrm{Xu}$, Yan fei Su and Yingjun Li \\ Inner Mongolia Electric Power (Group) Co., Ltd. Ulanchabu Electric Power BureauUlanchabu, China
}

\begin{abstract}
With the rapid development of local economy, the frequent occurrence of extreme climates, and the increase in the scale of new energy installations, short-term peak loads have repeatedly reached new highs, and the characteristics of the power system "double high, double peaks, and double-sided random" will be further highlighted, making the power grid supply and demand Balance faces greater challenges. In this context, the role of electricity demand response has become more prominent. Therefore, based on the principle of market elasticity, this paper studies the interactive response relationship between electricity price and load, establishes a demand response model based on price elasticity, and verifies demand response projects based on actual cases. The effect of peak clipping and valley filling.
\end{abstract}

\section{Introduction}

There according to the principles of economics, the elasticity of electricity market includes the self-elasticity of electricity and the cross-elasticity of electricity. The self-elasticity is used to measure the impact of current price changes in a single period on electricity demand, while the cross-elasticity is used to measure the impact of price changes in a multi-period on electricity demand. At present, domestic and foreign scholars have done a lot of research on demand response model based on market elasticity.

Taking into account the price elasticity of demand for consumer electrical equipment, literature [1] proposed a method for evaluating the price elasticity of residential electricity demand based on the characteristics of equipment power consumption. According to the definition of demand price elasticity, the use characteristics and self-elasticity of residential electrical equipment were analyzed. Cross-elastic correlation, combined with the classification of equipment adjustability associated with electricity consumption characteristics, constructs a residential electricity price response behavior analysis model based on equipment electricity adjustment fuzzy inference;Reference [2] focuses on how to automate and intelligentize the load on the user side Participate in demand response, design and implement a user-side automatic demand response system based on load decomposition to realize user participation in price-based demand response;Literature [3] studies the demand price elasticity matrix and proposes a method based on elasticity effect weights. Improve the price elasticity matrix model; in order to improve the operability of demand response potential evaluation; literature [4] proposed a quantitative evaluation method for demand response potential considering the statistical characteristics of load power consumption. This method is based on the principles of econometrics to obtain electricity demand-price elasticity Coefficient, quantify the electricity price level and the power demand under the incentive policy;literature [5] uses the user's demand price elasticity matrix for power commodities to establish a peak-valley time-of-use electricity price mathematical model;literature [6] establishes a reasonable time-of-use price and compensation Price, based on the electricity price elasticity coefficient, establishes an electricity demand response model, and uses the price signal to transfer part of the electricity consumption during the peak load period to the load trough period, achieving peak-shaving and valley-filling;literature [7] according to demand price elasticity Matrix (PEMD), proposes an improved PEMD model based on elastic effect weight; Literature [8] proposes a demand-side management evaluation model of distribution system based on the concept of elasticity and time-of-use pricing.

Research on the interactive relationship between load and electricity price based on market elasticity

Analysis of Electricity Market Elasticity

The price elasticity of electricity demand refers to the relative change in the amount of electricity demand caused by the relative change in the price of electricity, that is, the ratio of the rate of change of the user's electricity consumption to the rate of change of the corresponding price within a certain period of time. According to economic principles, a typical demand-price relationship curve is shown in Figure 1.

\footnotetext{
*Corresponding author: 595910434@qq.com
} 


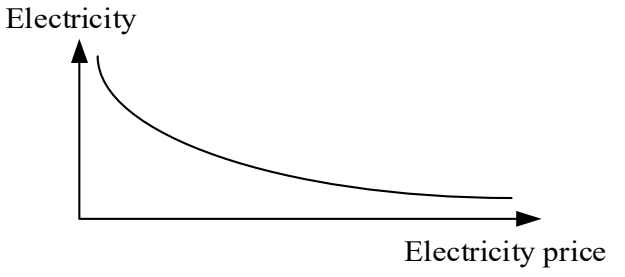

Figure 1. Typical demand price relationship curve

Under normal circumstances, when the price of electricity rises, the demand for electricity will decrease accordingly. The response to the electricity demand triggered by the electricity price, that is, the price elasticity of demand represented by the elastic coefficient, is shown in equation (1):

$$
\varepsilon=\frac{\Delta q}{q_{0}} \frac{p_{0}}{\Delta p}
$$

In the formula, $\Delta q$ and $\Delta p$ respectively represent the relative $q_{0}$ increase of the original load and the original $p_{0}$ electricity price.

Because in real life, the electricity demand of most users in a certain period of time is not only affected by the electricity price of that period, but also related to the electricity price of an adjacent time. In the multi-period response, the load elasticity coefficient can be divided into the self-elasticity coefficient and the cross elasticity coefficient according to the response time period. The self-elasticity coefficient represents the change in user load caused by the electricity price in the current period, and the cross-elasticity coefficient represents the impact of the electricity price in other periods on the user's electrical load. The coefficient of self-elasticity and crosselasticity are as follows:

$$
\begin{gathered}
\varepsilon_{i i}=\frac{\Delta q_{i}}{q_{i}} \frac{p_{i}}{\Delta p_{i}} \\
\varepsilon_{i j}=\frac{\Delta q_{i}}{q_{i}} \frac{p_{j}}{\Delta p_{j}}
\end{gathered}
$$

In the formula, $\varepsilon_{i i}$ represents the self-elasticity coefficient, $\varepsilon_{i j}$ represents the cross-elasticity coefficient, and the subscripts represent the $\mathrm{i}$-th and $\mathrm{j}$-th periods, respectively. From the self-elasticity coefficient and the cross-elasticity coefficient, the elasticity matrix E of electricity load and electricity price is obtained as follows:

$$
E=\left[\begin{array}{cccc}
\varepsilon_{11} & \varepsilon_{12} & \mathrm{~L} & \varepsilon_{1 n} \\
\varepsilon_{21} & \varepsilon_{22} & \mathrm{~L} & \varepsilon_{2 n} \\
\mathrm{M} & \mathrm{M} & & \mathrm{M} \\
\varepsilon_{n 1} & \varepsilon_{n 2} & \mathrm{~L} & \varepsilon_{n n}
\end{array}\right]
$$

The interactive relationship model between electricity load and electricity price
The interactive relationship model between electricity load and electricity price in the period $1 \sim n$ can be expressed as follows:

$\left[\begin{array}{c}\frac{\Delta q_{1}}{q_{1}} \\ \frac{\Delta q_{2}}{q_{2}} \\ \mathrm{M} \\ \frac{\Delta q_{n}}{q_{n}}\end{array}\right]=\left[\begin{array}{cccc}\varepsilon_{11} & \varepsilon_{12} & \mathrm{~L} & \varepsilon_{1 n} \\ \varepsilon_{21} & \varepsilon_{22} & \mathrm{~L} & \varepsilon_{2 n} \\ \mathrm{M} & \mathrm{M} & & \mathrm{M} \\ \varepsilon_{n 1} & \varepsilon_{n 2} & \mathrm{~L} & \varepsilon_{n n}\end{array}\right]\left[\begin{array}{c}\frac{\Delta p_{1}}{p_{1}} \\ \frac{\Delta p_{2}}{p_{2}} \\ \mathrm{M} \\ \frac{\Delta p_{n}}{p_{n}}\end{array}\right]=E\left[\begin{array}{c}\frac{\Delta p_{1}}{p_{1}} \\ \frac{\Delta p_{2}}{p_{2}} \\ \mathrm{M} \\ \frac{\Delta p_{n}}{p_{n}}\end{array}\right]$

Thus, the energy load of each period after the price-based demand response transformation can be obtained as:

$$
\begin{aligned}
q_{t}^{\prime} & =q_{t}+\Delta q_{t} \\
& =q_{t}\left[1+E(t, t) \frac{\Delta p_{t}}{p_{t}}+\sum_{\substack{i=1 \\
i \neq t}}^{n} E(t, i) \frac{\Delta p_{i}}{p_{i}}\right]
\end{aligned}
$$

Then the load change in this period is:

$$
\Delta q_{t}=q_{t}\left[E(t, t) \frac{\Delta p_{t}}{p_{t}}+\sum_{\substack{i=1 \\ i \neq t}}^{n} E(t, i) \frac{\Delta p_{i}}{p_{i}}\right]
$$

According to the current situation of demand response in various provinces and cities in China and the research experience of many scholars, the price elasticity coefficient of demand during peak, peak, flat, and valley periods can be obtained as shown in Table 2-1. The selfelasticity coefficient of electricity demand price during valley period is -0.15 , Which shows that the change in electricity price during the valley period is negatively correlated with the change in the electricity demand during that period, and when the electricity price in the valley period increases by $1 \%$, the user's power demand in the valley period decreases by $0.15 \%$. The cross elasticity coefficient of the electricity demand price during the normal period to the valley period is 0.03 , indicating that the change in electricity price during the normal period is positively correlated with the change in the electricity demand during the valley period, and when the electricity price in the normal period increases by $1 \%$, the user's power demand in the valley period decreases by $0.03 \%$.

Table 1. Price dlasticity coefficient of electricity demand by users

\begin{tabular}{ccccc}
\hline$\varepsilon$ & Valley & flat & peak & sharp peak \\
\hline Valley & -0.15 & 0.03 & 0.06 & 0.04 \\
flat & 0.03 & -0.15 & 0.02 & 0.06 \\
peak & 0.06 & 0.02 & -0.15 & 0.03 \\
sharp peak & 0.04 & 0.06 & 0.03 & -0.15 \\
\hline
\end{tabular}




\section{Example analysis}

This section selects the time-of-use electricity price before the implementation of the demand response and the peak electricity price after the implementation of the demand response in a province, as well as the forecasted load data of a typical summer day in 2025 (as shown in Figures 2 and 3).

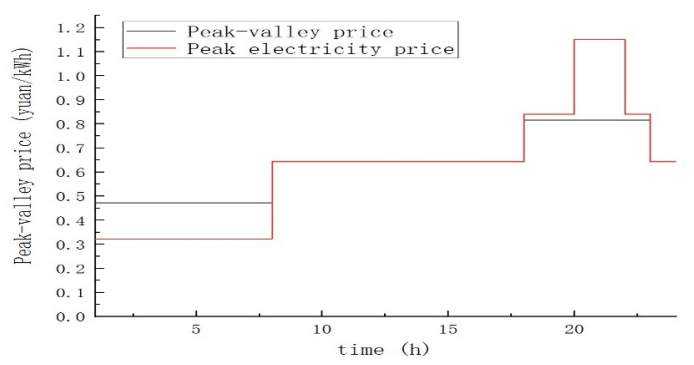

Figure 2. Electricity price before and after the implementation of demand response

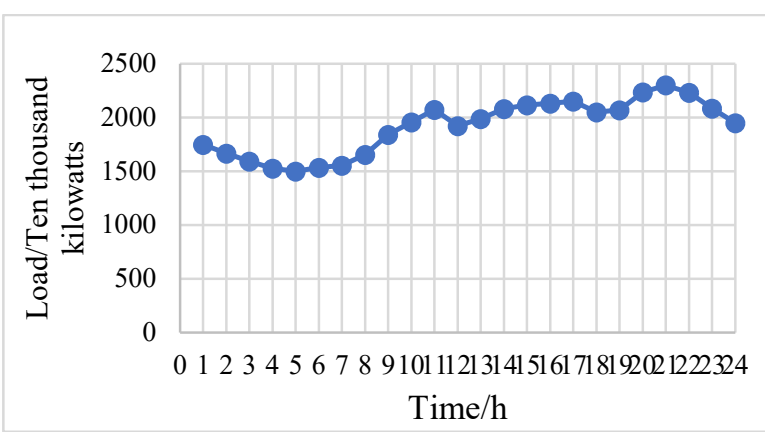

Figure 3. Typical daily load curve in the summer of 2025

The interactive relationship model between electricity load and electricity price in time period 1 24 is calculated as follows:

$$
\left[\begin{array}{c}
\frac{\Delta q_{1}}{q_{1}} \\
\frac{\Delta q_{2}}{q_{2}} \\
\mathrm{M} \\
\frac{\Delta q_{24}}{q_{24}}
\end{array}\right]=\left[\begin{array}{cccc}
\varepsilon_{11} & \varepsilon_{12} & \mathrm{~L} & \varepsilon_{1,24} \\
\varepsilon_{21} & \varepsilon_{22} & \mathrm{~L} & \varepsilon_{2,24} \\
\mathrm{M} & \mathrm{M} & & \mathrm{M} \\
\varepsilon_{24,1} & \varepsilon_{24,2} & \mathrm{~L} & \varepsilon_{24,24}
\end{array}\right]\left[\begin{array}{c}
\frac{\Delta p_{1}}{p_{1}} \\
\frac{\Delta p_{2}}{p_{2}} \\
\mathrm{M} \\
\frac{\Delta p_{24}}{p_{24}}
\end{array}\right]=E\left[\begin{array}{c}
\frac{0.4759625-0.47179}{0.47179} \\
\frac{0.47179}{\mathrm{M}} \\
\frac{0.4759625-0.47179}{0.64371}
\end{array}\right]=\left[\begin{array}{c}
0.02768352 \\
0.02768352 \\
\mathrm{M} \\
0.0024118
\end{array}\right]
$$

Assuming that users who implement time-of-use electricity prices account for $50 \%$, and users who do not implement time-of-use electricity prices account for $50 \%$, from equation (8), the energy load of each period after the price-based demand response transformation can be obtained as:

$$
\begin{aligned}
q_{t}^{\prime} & =q_{t}+0.5 q_{t} \frac{\Delta q_{t}}{q_{t}} \\
& =\left[\begin{array}{c}
1749 \\
1665 \\
\mathrm{M} \\
1948
\end{array}\right]+0.5\left[\begin{array}{c}
1749 \times 0.02768352 \\
1665 \times 0.02768352 \\
\mathrm{M} \\
1948 \times 0.0024118
\end{array}\right]=\left[\begin{array}{c}
1773 \\
1688 \\
\mathrm{M} \\
1995
\end{array}\right]
\end{aligned}
$$

Specifically, according to formula $(1) \sim$ formula $(9)$ to calculate the load of each period after the implementation of the time-of-use electricity price, as shown in Table2. and Figure4.shown.

\begin{tabular}{|c|c|c|c|c|}
\hline \multicolumn{2}{|c|}{ Time period } & $\begin{array}{l}\text { Original } \\
\text { load(Ten } \\
\text { thousand } \\
\text { kilowatt) }\end{array}$ & $\begin{array}{l}\text { Load after } \\
\text { implementation(Ten } \\
\text { thousand kilowatts) }\end{array}$ & $\begin{array}{c}\text { load } \\
\text { change(Ten } \\
\text { thousand } \\
\text { kilowatts) }\end{array}$ \\
\hline \multirow{8}{*}{ Valley } & 1 & 1749 & 1773 & 24 \\
\hline & 2 & 1665 & 1688 & 23 \\
\hline & 3 & 1591 & 1613 & 22 \\
\hline & 4 & 1526 & 1548 & 22 \\
\hline & 5 & 1500 & 1521 & 21 \\
\hline & 6 & 1535 & 1556 & 21 \\
\hline & 7 & 1553 & 1575 & 22 \\
\hline & 8 & 1656 & 1679 & 23 \\
\hline \multirow{10}{*}{ flat } & 9 & 1842 & 1886 & 44 \\
\hline & 10 & 1956 & 2003 & 47 \\
\hline & 11 & 2073 & 2123 & 50 \\
\hline & 12 & 1923 & 1969 & 46 \\
\hline & 13 & 1989 & 2036 & 47 \\
\hline & 14 & 2079 & 2129 & 50 \\
\hline & 15 & 2114 & 2164 & 50 \\
\hline & 16 & 2131 & 2182 & 51 \\
\hline & 17 & 2152 & 2203 & 51 \\
\hline & 18 & 2049 & 2098 & 49 \\
\hline \multirow{2}{*}{ peak } & 19 & 2068 & 2083 & 15 \\
\hline & 20 & 2234 & 2250 & 16 \\
\hline sharp & 21 & 2300 & 2171 & -129 \\
\hline peak & 22 & 2230 & 2104 & -126 \\
\hline peak & 23 & 2084 & 2099 & 15 \\
\hline flat & 24 & 1948 & 1995 & 47 \\
\hline
\end{tabular}

Table 2. Loads in each period after the implementation of time-of-use electricity

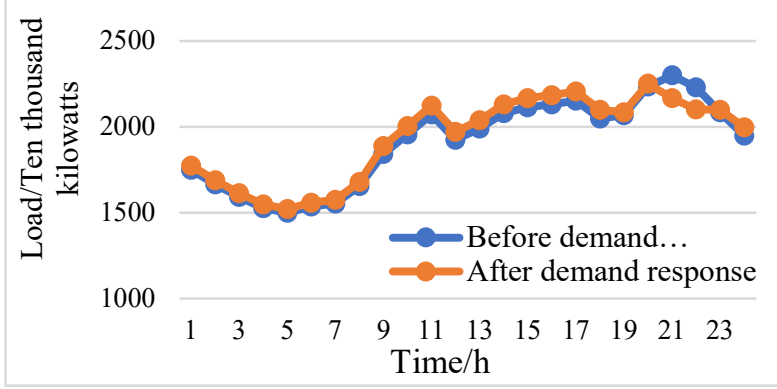

Figure 4. Changes in load in each period before and after the implementation of time-of-use electricity prices

According to Figure 4, the implementation of time-of-use electricity prices can achieve the effect of peak shaving and valley filling. When the peak-to-valley price difference is 0.665 yuan, a total of 2.55 million kilowatts of load will be transferred during peak periods to flatvalley periods, and the total load during valley periods will increase by 1.78 million kilowatts. The peak cut percentage of the highest load before the implementation of time-of-use electricity prices can reach $5.61 \%$. The percentage of grain filling at the lowest load reached $1.40 \%$.

\section{Conclusion}

From this study, the implementation of time-of-use electricity prices has played a role in peak-shaving and valley-filling. When the peak electricity price is raised by 
0.01 yuan, the peak-to-valley price difference increases to 0.675 yuan. During the peak period, a total of 2.63 million kilowatts of load is transferred to the flat valley period. The load during the valley period increases by 1.83 million kilowatts, and the peak cut percentage of the highest load can reach $5.81 \%$, The percentage of grain filling at the lowest load reached $1.43 \%$. When the peak electricity price was increased by 0.02 yuan, the peak-tovalley price difference reached 0.685 yuan. During the peak period, a total of 2.71 million kilowatts of load was transferred to the flat valley period. The load during the valley period increased by 1.89 million kilowatts. The peak load of the highest load before the time-of-use electricity price was implemented. The percentage can reach $5.99 \%$, and the percentage of valley filling with the lowest load reaches $1.48 \%$. Therefore, increasing the peak-to-valley spread can improve the peak-shaving and valley-filling effect.

\section{Acknowledgments}

This work was financially supported by Science and Technology Project of Inner Mongolia Electric Power Group (Limited) Company (WD-ZXZB-2021-SC04020304): Research on Key Technologies of Coordinated Control of Ulanqab Regional Power System.

\section{References}

1. Xu Guangda, Zhang Li, Liang Jun, Sun Donglei, Zhao Long, Han Xueshan. Evaluation of residential electricity demand price elasticity based on equipment power consumption characteristics $[\mathrm{J}]$. Automation of Electric Power Systems, 2020, 44(13): 48-55.

2. Sun Zhiqing, Wang Shouxiang, Zhou Kai, Liu Tianyu. User-side automatic demand response system based on load decomposition[J]. Journal of Electric Power System and Automation, 2016, 28(12): 64-69+88.

3. Liu Haotian, Chen Jin, Zhu Xi, et al. A peak-valley time-of-use electricity price incentive strategy for residents based on price elasticity matrix [J]. Power System Protection and Control, 2021, 49(5): 116-123. DOI: 10.19783/j.cnki.pspc.200527.

4. Li Zhangyun, Wang Gang, Ding Maosheng, et al. Demand response potential evaluation considering the statistical characteristics of load power consumption[J]. Chinese Science and Technology Paper, 2017, 12(5): 529-536. DOI:10.3969/j. issn.2095-2783.2017.05.010.

5. Sun Wei, Li Shan. Research on time-of-use electricity price in power demand side management [J]. Electrical Application, 2008,27(6):63-66. DOI:10.3969/j.issn.1672-9560.2008.06.019.

6. Sun Qian, Peng Jianchun, Pan Juntao, et al. Congestion management for multi-period power demand response[J]. Power System Technology, 2010, 34(9): 139-143.
7. Wang Lei, He Jianjian, Zhou Mei, et al. Research on multi-frequency control of power grid peak-valley time-sharing load based on demand response[J]. Electronic Design Engineering, 2020, 28(11): 5-8, 13. DOI: 10.14022 /j.issn1674-6236.2020.11.002.

8. Juan Carlos Galvis, Agostino Costa. Demand Side Management Using Time of Use and Elasticity Price[J]. IEEE Latin America Transactions, 2016,14(10): 